\title{
BMJ Open Non-invasive brain stimulation interventions for management of chronic central neuropathic pain: a scoping review protocol
}

\author{
Mei Lin Chen, ${ }^{1}$ Lin Yao, ${ }^{1}$ Jennifer Boger, ${ }^{1}$ Kathryn Mercer, ${ }^{2}$ Benjamin Thompson, ${ }^{3}$
} Ning Jiang ${ }^{1}$

To cite: Chen ML, Yao L, Boger J, et al. Non-invasive brain stimulation interventions for management of chronic central neuropathic pain: a scoping review protocol. BMJ Open 2017;7:e016002. doi:10.1136/ bmjopen-2017-016002

- Prepublication history for this paper is available online. To view these files, please visit the journal online (http://dx.doi org/10.1136/bmjopen-2017016002).

Received 20 January 2017 Revised 6 September 2017 Accepted 7 September 2017

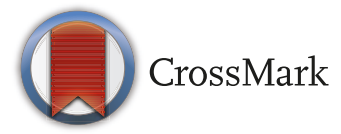

${ }^{1}$ Department of Systems Design Engineering, Faculty of Engineering, University of Waterloo, Waterloo, Canada ${ }^{2}$ School of Pharmacy, University of Waterloo, Waterloo, Canada ${ }^{3}$ Department of Optometry and Vision Science, University of Waterloo, Waterloo, Canada

Correspondence to

Dr Ning Jiang;

ning.jiang@uwaterloo.ca

\section{ABSTRACT}

Introduction Pain can affect people regardless of age, gender or ethnicity. Chronic central neuropathic pain (CCNP) is a debilitating condition that affects populations such as stroke survivors, amputees, spinal cord injury patients and patients with multiple sclerosis, with prevalence rates between $30 \%$ and $80 \%$. This condition can be caused by a lesion or disease affecting the somatosensory system. CCNP is notoriously drug resistant, and few effective CCNP treatment or management strategies exist. The emergence of non-invasive brain stimulation and neuromodulation techniques provide novel avenues for managing chronic central neuropathic pain. This scoping review aims to systematically identify the methods and effectiveness of non-invasive brain stimulation techniques for treating and managing chronic central neuropathic pain.

Methods and analysis The following databases will be searched systematically: PubMed, EMBASE, Cumulative Index to Nursing and Allied Health Literature (CINAHL), Institute of Electric and Electronic Engineers (IEEE), Association of Computing Machinary (ACM) and Scopus. Additional literature will be identified by searching the reference lists of identified studies. Studies will include reviews and original research in both published and grey literatures. Two reviewers will independently screen identified studies for final inclusion. A quantitative analysis on the intervention type, application and efficacy will be synthesised along with a qualitative analysis to describe the effectiveness of each intervention.

Ethics and dissemination № primary data will be collected and hence formal ethics review is not required. The results of the scoping review will be presented at relevant national and international conferences, published in a peer-reviewed journal and provided to the stakeholders with plain language to be posted on their websites. This scoping review will provide a foundation to guide the development of future primary research on noninvasive brain stimulation and CCNP.

\section{INTRODUCTION}

Pain is a critical mechanism for harm prevention. However, the experience of chronic pain can dramatically reduce one's quality
Strengths and limitations of this study

- This study protocol provides an overview of the current status of the field to inform the development of a scoping review of non-invasive brain stimulation interventions for the management of chronic central neuropathic pain.

- The scoping review will identify gaps in research and in the translation of research results into clinical practice.

- The scoping review is specific to active stimulation interventions, excluding pharmacotherapy interventions.

- A limitation is that this scoping review that aims to explore the field for existing methods rather than a systematic review that seeks to answer a specific question.

of life, affecting the individual, their families and society at large. ${ }^{12}$ Pain is defined by the International Association for the Study of Pain (IASP) as: 'an unpleasant sensory and emotional experience associated with actual or potential tissue damage, or described in terms of such damage'. ${ }^{3}$ Specifically, the IASP defined neuropathic pain as 'pain caused by a lesion or disease of the somatosensory nervous system'. ${ }^{3}$ Neuropathic pain is a clinical description (and not a diagnosis) that requires a demonstrable lesion or a disease that satisfies established neurological diagnostic criteria. ${ }^{3}$ The term lesion is commonly used in diagnostic investigations (eg, imaging, neurophysiology, biopsies and lab tests) to reveal an abnormality or obvious trauma. The term disease is commonly used when the underlying cause of the lesion is known (eg, stroke, vasculitis, diabetes mellitus and genetic abnormality). Somatosensory refers to information about the body including visceral organs. ${ }^{3}$ 
The burden of disability associated with neuropathic pain is substantial and includes healthcare costs, time and quality of life. ${ }^{4}$ The health-related quality of life for people with neuropathic pain is comparable with cancer or chronic heart failure. ${ }^{5}$ In Canada, chronic pain involves healthcare costs that exceed $\$ 6$ billion per year and productivity costs at $\$ 37$ billion per year. ${ }^{6}$ Furthermore, an analysis of a large US insurance database revealed that healthcare costs of patients with neuropathic pain were three times greater than those of age-matched and sex-matched claimants without neuropathic pain. ${ }^{7}$

Currently, there exists no treatment to prevent the development of neuropathic pain following injury to the somatosensory system, neither is there a method to specifically control the pain when it is established. Pharmacotherapy for neuropathic pain has been generally disappointing ${ }^{8-11}$; patients with neuropathic pain do not respond well to non-steroidal anti-inflammatory drugs, and resistance or insensitivity to opiates is common. ${ }^{21213}$ Examples of pharmacological treatments include tricyclic or serotonin and norepinephrine uptake inhibitors, antidepressants and anticonvulsants, which all have limited efficacy and undesirable side effects. ${ }^{28}$ Ancillary treatments such as physical and psychological interventions are often used to help patients cope with pain, although they are less effective for severe pain. ${ }^{9}$

Alternatively, a number of invasive and non-invasive stimulation techniques are increasingly being proposed either as a substitute for or in combination with current medical therapies. ${ }^{9}$ Peripheral stimulation techniques include: transcutaneous electrical nerve stimulation (TENS) and nerve root stimulation (NRS). TENS involves surface electrodes placed over the painful area or its associated nerve, delivers stimulation at high frequency and low intensity to activate the $\mathrm{A} \beta$ afferents and evokes paraesthesia in the pain area, providing short-term (20-30 min) pain relief in accordance with the gate-control theory. ${ }^{9} \mathrm{NRS}$ involves implantation of an electrode in the root exit from the spine. These peripheral stimulation techniques provide only temporary pain relief that lasts for the duration of the stimulation. ${ }^{9}$ Other invasive stimulation techniques include: spinal cord stimulation (SCS), deep brain stimulation (DBS) and motor cortex stimulation (MCS). These procedures involve the implantation of a stimulating device into their respective target areas: SCS targets the posterior thoracic space of the thoracic or cervical spine, DBS targets the sensory thalamus or periventricular grey matter and MCS targets the motor cortex. The effectiveness of these invasive techniques varies significantly across patients ${ }^{14}$ and their mechanisms of action are unclear. ${ }^{15}$ Furthermore, the techniques are only applicable to patients who can safely undergo surgery. ${ }^{9}$

Examples of non-invasive brain stimulation techniques include: transcranial direct-current stimulation (tDCS),${ }^{1416}$ and repetitive transcranial magnetic stimulation (rTMS). ${ }^{91718}$ Both stimulation techniques are adjustable and reversible. ${ }^{9}$ tDCS has shown promising results in inducing cortical-plasticity with clinical benefits. ${ }^{19}$ It passes a weak (commonly $\leq 2 \mathrm{~mA}$ ) monophasic electric current to the cerebral cortex through the scalp, modifying neuron membrane excitability, leading to neuroplasticity. ${ }^{19} \mathrm{~A}$ single tDCS session can induce transient cortical effects, but daily sessions may induce longer lasting effects. ${ }^{19}$ For rTMS, the stimulation is applied using a magnetic stimulation coil placed against the head. A rapidly changing magnetic field is induced in the coil that generates an electrical current in the cortical area below the coil. rTMS has been found to affect neuropathic pain processing. ${ }^{9} 2$ The clinical effects are modest and short-lasting from a single session, ${ }^{9}$ but repeated sessions may cause greater and longer lasting effects. There exists equivocal evidence and differing perspectives on the effects of rTMS; for instance, one study suggests that rTMS can produce longlasting pain relief, ${ }^{17}$ while another states that its effects are uncertain and may be mediated by other factors such as mood..$^{21}$ Given that non-invasive brain stimulation may relieve neuropathic pain, and the effect size is modest, there is significant heterogeneity between studies that should be further investigated. ${ }^{22}$

There is a need for a systematic overview of the existing evidence to support further research.

Previous reviews have been done to gather the research evidence on isolated topics and NIBS techniques for specific conditions. However, a broad scoping review with a clear search strategy is needed to scope the wide-ranging evidence for non-invasive brain stimulation techniques for chronic central neuropathic pain. This scoping review will fill this gap by summarising the breadth, depth and clinical applications of current non-invasive brain stimulation interventions for chronic central neuropathic pain, distilling the existing research to support the future development of primary research. ${ }^{23} 24$

\section{AIM}

This article describes a protocol for a scoping review that will locate, summarise and report literature that informs the current and proposed non-invasive brain stimulation interventions for chronic central neuropathic pain, as well as identify areas to direct future research.

The scoping review will:

1. review the breadth and depth of peer-reviewed literature that has examined or evaluated the application of non-invasive brain stimulation techniques to chronic central neuropathic pain in technology and medical databases;

2. review the extent and nature of a sampling of non-peer-reviewed non-invasive brain stimulation interventions for chronic central neuropathic pain from key organisational websites, professional regulatory bodies and special interest organisations and disease-specific groups.

\section{METHODS AND ANALYSIS}

Our scoping team of reviewers will be multidisciplinary, comprising of clinician-researchers, engineering researchers and health researchers. The scoping review 
methodology laid out by Arksey and O'Malley ${ }^{24}$ and further clarified by Levac et $a l^{23}$ will be used. The approach will review the existing literature and provide transparency, reproducibility and utility within this protocol. ${ }^{25}$

\section{Identifying the research question}

The research question was framed by assimilating themes from preliminary searches, and opinions were sought from experts in the field of pain rehabilitation and neurotechnology. Using a concept, target population and outcomes of interest approach, we formulated a broad research question: 'What are the nature, adherence, extent, efficacy, exposure, quality of delivery and clinical application of non-invasive brain stimulation techniques currently used and proposed in managing chronic central neuropathic pain?'.

\section{Eligibility criteria}

The following inclusion criteria were used to guide the search and will be used when reviewing articles:

- published in the English language

- human subjects with chronic central neuropathic pain

- years of publication: none specified

- all age groups

- articles that include at least one non-invasive brain stimulation intervention.

\section{Exclusion criteria}

- Commentaries

- editorials

- narrative reviews

- books and book chapters

- lectures and addresses

- animal studies.

\section{Types of study}

Meta-analysis, systematic reviews, randomised control trials, cohort studies, case-control studies, case series/ case reports and cross-sectional trials.

\section{Databases}

Published studies will be identified from the following electronic database: PubMed, Embase, Cumulative Index to Nursing and Allied Health Literature, IEEE, ACM and Scopus. Additional literature will be identified by hand searching the reference list of identified eligible studies and as well through identified grey literature sources.

\section{Search strategy}

The in-depth search strategy has been developed in each of the six databases to capture the broad literature on the topic. In order to maximise the sensitivity of the search, the following steps will be taken: consult with experts in the field; search in clinical trials registers, conference proceedings and selected grey literature such as $\mathrm{PhD}$ theses; perform forward and backward citation tracking; contact the websites of key organisations; and handsearch journal references.
Please see a sample search strategy in PubMed as follows:

('neuralgia' [MeSH Terms] OR 'neuralgia'[All Fields] OR ('neuropathic'[All Fields] AND 'pain'[All Fields]) OR 'neuropathic pain' [All Fields]) AND ('therapy' [Subheading] OR 'therapy'[All Fields] OR 'treatment'[All Fields] OR 'therapeutics' [MeSH Terms] OR 'therapeutics'[All Fields]) OR ('Brain Stimul'[Journal] OR ('brain'[All Fields] AND 'stimulation'[All Fields]) OR 'brain stimulation' [All Fields]) OR (non[All Fields] AND invasive[All Fields]) AND (Clinical Trial[ptyp] AND 'humans' [MeSH Terms] AND English[lang]).

\section{Study selection}

Study screening will be reported and guided according to Levac et al s framework and the reporting will follow the Preferred Reporting Items for Systematics Reviews and Meta-analyses checklist and will be performed in four major stages ${ }^{26}$ First, search results will be merged and duplicates will be removed via reference management software (EndNote V.X5). Second, a data extraction form based on the eligibility criteria described above will be developed by the research team. Third, a pilot test of this data extraction form will be performed: two reviewers will independently screen the first 25 titles, abstracts and grey literature of retrieved publications according to the eligibility criteria by using the data extraction form. Fourth, all eligible studies and those classified as unclear (needing more information) will be reviewed in full-text by each reviewer independently to determine if all inclusion criteria are met and if the article is to be included in the study. Inter-rater agreement will again be calculated on a random sample of 25 articles. Disagreement on study eligibility will be discussed and resolved with a third reviewer.

\section{Data extraction}

A customised data extraction form will be constructed to extract all relevant data from each study. Two reviewers will use the form to extract data from the first 15 eligible articles. Then they will meet to compare consistency of data extraction and coding. Clarification and updating of the extraction form will be an iterative process until all authors reach consensus on the final version. The data extraction form will be piloted on the first five eligible studies to evaluate its reliability in capturing the study data of interest. Data extraction will be undertaken independently by two reviewers.

Descriptive summary tables will be produced to recapitulate the evidence base. The following data will be extracted:

- author(s) and date

- geographical location

- research design

- aim

- research question

- methods

- settings 
- participant characteristics (total number, mean age, gender, pathology if available)

- primary cause of pain

- pain characteristics

- intervention studied/proposed

- intervention rationale/mechanism

- intervention frequency, site, duration of stimulation and the delay between times of stimulation and the clinical effects

- attitude towards intervention (positive or negative feelings towards intervention-from the healthcare provider; positive or negative feelings towards treatment-from the participant/patient and so on)

- NIBS characteristic (intensity, number of pulses, montage, current pattern/waveform and duration)

- length of follow-up

- duration of effect

- patient response, participation and enthusiasm in intervention

- simultaneous interventions (if applicable)

- neuropathic pain comorbidities

- key findings

- research gaps identified

- Potential biases in study (assessed using the Cochrane Collaboration's 'Risk of bias' tool for assessing risk of bias) $)^{26}$

- pain outcome (visual analogue scale (VAS) if available)

- daily functioning outcome.

Other variables may be added when revising and updating the data extraction form after analysis of the first 15 eligible articles.

\section{Data synthesis}

An initial map will be developed to explore the interventions available for chronic central neuropathic pain. The findings will be quantitatively and qualitatively synthesised for all identified interventions. The quantitative synthesis will comprise of numerical counts such as number of interventions by setting and by application. A qualitative description approach will be used to describe the characteristics of each intervention (ie, definition of intervention, mechanism, efficacy, side effects, frequency of use and feasibility) as well as pain outcome using the VAS. The change in VAS, for example, will be used to review the effectiveness of the intervention. The specific metrics that will be included will be determined after the papers have been identified and reviewed.

\section{ETHICS AND DISSEMINATION}

There are no protected/private health information collected, hence there will be no need for formal ethical review. The results of the scoping review will be presented at relevant national and international conferences, published in a peer-reviewed journal and proposed to relevant stakeholders.
CONCLUSION

This scoping review will map key concepts and empirical results relating to the use of non-invasive brain stimulation to treat and manage chronic central neuropathic pain. It will also provide a comprehensive evaluation of current methodologies and identify gaps for future research and share the key research findings with relevant stakeholders.

Contributors Designed the scoping review protocol: MLC and KM. Performed literature search: MLC, LY, KM, BT and NJ. Contributed analysis tools: LY, JB, BT and NJ. Wrote the paper: MLC Proofread manuscript: all authors.

Funding This work was supported by the University Starter Grant of the University of Waterloo (\#203859). This research received no specific grant from any funding agency in the public, commercial or not-for-profit sectors. We would also like to thank Dannie Fu, a current member of the research lab, for her assistance in proofreading and editing.

Competing interests None declared.

Provenance and peer review Not commissioned; externally peer reviewed.

Open Access This is an Open Access article distributed in accordance with the Creative Commons Attribution Non Commercial (CC BY-NC 4.0) license, which permits others to distribute, remix, adapt, build upon this work non-commercially, and license their derivative works on different terms, provided the original work is properly cited and the use is non-commercial. See: http://creativecommons.org/ licenses/by-nc/4.0/

(C) Article author(s) (or their employer(s) unless otherwise stated in the text of the article) 2017. All rights reserved. No commercial use is permitted unless otherwise expressly granted.

\section{REFERENCES}

1. Reitsma ML, Tranmer JE, Buchanan DM, et al. The prevalence of chronic pain and pain-related interference in the Canadian population from 1994 to 2008. Chronic Dis Inj Can 2011;31:157-65.

2. Woolf CJ, Mannion RJ. Neuropathic pain: aetiology, symptoms, mechanisms, and management. Lancet 1999;353:1959-64.

3. International Association for the Study of Pain, "IASP Taxonomy,". 2012 https://www.iasp-pain.org/Education/Content.aspx? ItemNumber $=1698 \&$ navltemNumber $=576 \#$ Pain (accessed $04 \mathrm{Jul}$ 2017).

4. Schopflocher D, Taenzer P, Jovey R. The prevalence of chronic pain in Canada. Pain Res Manag 2011;16:445-50.

5. Meyer-Rosberg K, Burckhardt CS, Huizar K, et al. A comparison of the SF-36 and Nottingham Health Profile in patients with chronic neuropathic pain. Eur J Pain 2001;5:391-403.

6. Lynch ME. The need for a Canadian pain strategy. Pain Res Manag 2011;16:77-80.

7. Berger A, Dukes EM, Oster G. Clinical characteristics and economic costs of patients with painful neuropathic disorders. J Pain 2004;5:143-9.

8. Finnerup NB, Attal N, Haroutounian S, et al. Pharmacotherapy for neuropathic pain in adults: a systematic review and meta-analysis. Lancet Neurol 2015;14:162-73.

9. Cruccu G, Aziz TZ, Garcia-Larrea L, et al. EFNS guidelines on neurostimulation therapy for neuropathic pain. Eur $\mathrm{J}$ Neurol 2007:14:952-70.

10. Finnerup NB, Otto M, McQuay HJ, et al. Algorithm for neuropathic pain treatment: an evidence based proposal. Pain 2005;118:289-305.

11. Mulla SM, Wang L, Khokhar R, et al. Management of Central Poststroke Pain. Stroke 2015;46:2853-60.

12. Arnér S, Meyerson BA. Lack of analgesic effect of opioids on neuropathic and idiopathic forms of pain. Pain 1988;33:11-23.

13. Portenoy RK, Foley KM, Inturrisi CE. The nature of opioid responsiveness and its implications for neuropathic pain: new hypotheses derived from studies of opioid infusions. Pain 1990;43:273-86.

14. Lefaucheur JP, Antal A, Ahdab R, et al. The use of repetitive transcranial magnetic stimulation (rTMS) and transcranial direct current stimulation (tDCS) to relieve pain. Brain Stimul 2008;1:337-44. 
15. Papuc E, Rejdak K. "The role of neurostimulation in the treatment of neuropathic pain,". Ann agric Env. med 2013;1:14-17.

16. Fregni F, Boggio PS, Lima MC, et al. A sham-controlled, phase II tria of transcranial direct current stimulation for the treatment of central pain in traumatic spinal cord injury. Pain 2006;122:197-209.

17. Khedr EM, Kotb H, Kamel NF, et al. Longlasting antalgic effects of daily sessions of repetitive transcranial magnetic stimulation in central and peripheral neuropathic pain. $J$ Neurol Neurosurg Psychiatry 2005;76:833-8.

18. Leo RJ, Latif T. Repetitive transcranial magnetic stimulation (rTMS) in experimentally induced and chronic neuropathic pain: a review. $J$ Pain 2007;8:453-9.

19. Souto G, Borges IC, Goes BT, et al. Effects of tDCS-induced motor cortex modulation on pain in HTLV-1: a blind randomized clinical trial. Clin J Pain 2014;30:809-15.

20. N. E. O'Connell, Wand BM, Marston L, et al. "Non-invasive brain stimulation techniques for chronic pain,". Cochrane Database of
Systematic Reviews no.4. Chichester, UK: John Wiley \& Sons, Ltd, 2014.

21. Ohn SH, Chang WH, Park $\mathrm{CH}$, et al. Neural correlates of the antinociceptive effects of repetitive transcranial magnetic stimulation on central pain after stroke. Neurorehabil Neural Repair 2012;26:344-52.

22. Lefaucheur JP. Cortical neurostimulation for neuropathic pain: state of the art and perspectives. Pain 2016;157:S81-S89.

23. Levac D, Colquhoun H, O'Brien KK. Scoping studies: advancing the methodology. Implement Sci 2010:5:69.

24. Arksey H, O'Malley L. Scoping studies: towards a methodological framework. Int J Soc Res Methodol 2005;8:19-32.

25. Armstrong R, Hall BJ, Doyle J, et al. 'Scoping the scope' of a cochrane review. J Public Health 2011;33:147-50.

26. Higgins J, Green S. Cochrane Handbook for Systematic Reviews of Interventions Version 5.1.0: The Cochrane Collaboration, 2011. (Updated Mar 2011). 